\title{
A NOTE ON THE EISENSTEIN ELEMENTS OF PRIME SQUARE LEVEL
}

\author{
DEBARGHA BANERJEE
}

(Communicated by Lev Borisov)

\begin{abstract}
We explicitly write the Eisenstein elements inside the space of modular symbols corresponding to each Eisenstein series for the congruence subgroup $\Gamma_{0}\left(p^{2}\right)$, answering a question of Merel. As a consequence, we also write the winding element explicitly for the congruence subgroup $\Gamma_{0}\left(p^{2}\right)$.
\end{abstract}

\section{INTRODUCTION}

For all $N \geq 1$, the congruence subgroup $\Gamma_{0}(N)$ is the subgroup of $\mathrm{SL}_{2}(\mathbb{Z})$ consisting of all matrices $\left(\begin{array}{ll}a & b \\ c & d\end{array}\right)$ such that $N \mid c$. This subgroup acts on the upper half plane $\mathbb{H}=\{z \in \mathbb{C} \mid \mathfrak{I m}(z)>0\}$ in the usual way. The quotient space $\Gamma_{0}(N) \backslash \mathbb{H}$ is denoted by $Y_{0}(N)$. Let $X_{0}(N)$ be the compactification of $Y_{0}(N)$ obtained by adjoining the set of cusps $\Gamma_{0}(N) \backslash \mathbb{P}^{1}(\mathbb{Q})$. Let $E_{2}\left(\Gamma_{0}(N)\right)$ be the space of weight 2 Eisenstein series for this congruence subgroup. Suppose the Fourier coefficients at $\infty$ of an Eisenstein series $E$ are in a fixed number field $K$. Let $\pi_{E}: \mathrm{H}_{1}\left(Y_{0}(N), K\right) \rightarrow K$ be the "period" homomorphism of $E$ as in Section [3.1. The intersection pairing $\circ$ [4], [2, p. 2] induces a perfect, bilinear pairing of $K$-vector spaces

$$
\mathrm{H}_{1}\left(X_{0}(N) \text {, cusps, } K\right) \times \mathrm{H}_{1}\left(Y_{0}(N), K\right) \rightarrow K \text {. }
$$

Since $\circ$ is a non-degenerate bilinear pairing, there is a unique element $\mathcal{E}$ such that $\mathcal{E} \circ c=\pi_{E}(c)$. The modular symbol $\mathcal{E}$ is the Eisenstein element corresponding to the Eisenstein series $E$.

Let us fix a prime $p \geq 3$. For the congruence subgroup $\Gamma_{0}(p)$, the Eisenstein element is well known by the fundamental work of Merel [9]. In this paper, we write the Eisenstein elements inside the space of modular symbols for the congruence subgroup $\Gamma_{0}\left(p^{2}\right)$. To our knowledge, this article is the first attempt to write the Eisenstein elements and winding element explicitly for the non-prime level. We hope to generalize our main theorem to any congruence subgroup of odd level (cf. Remark 4.6 and 4.8).

Let $\zeta: \mathrm{SL}_{2}(\mathbb{Z}) \rightarrow \mathrm{H}_{1}\left(X_{0}\left(p^{2}\right)\right.$, cusps, $\left.\mathbb{Z}\right)$ be the map that takes the matrix $g \in$ $\mathrm{SL}_{2}(\mathbb{Z})$ to the class in $\mathrm{H}_{1}\left(X_{0}\left(p^{2}\right)\right.$, cusps, $\left.\mathbb{Z}\right)$ of the image in $X_{0}\left(p^{2}\right)$ of the geodesic in $\mathbb{H} \cup \mathbb{P}^{1}(\mathbb{Q})$ joining $g .0$ and $g . \infty$. The map $\zeta$ is surjective (Manin [6]). If $x \in$ $\mathbb{P}^{1}\left(\mathbb{Z} / p^{2} \mathbb{Z}\right)$ is not of the form $(k p \pm 1,1)$ or $(1, k p \pm 1)$, then we write $x$ as $(r-1, r+1)$

Received by the editors December 21, 2011 and, in revised form, October 11, 2012 and November $15,2012$.

2010 Mathematics Subject Classification. Primary 11F67; Secondary 11F11, 11F20, 11F30.

Key words and phrases. Eisenstein series, modular symbols, special values of $L$-functions.

The author was partially supported by the ARC grant DP0773301. 
and let $\alpha_{r}, \beta_{r}$ be the matrices $\left(\begin{array}{cc}1 & -r \\ 0 & 2 p^{2}\end{array}\right),\left(\begin{array}{cc}1 & -r \\ 0 & p^{2}\end{array}\right)$. Let $\delta_{r}$ be 1 or 0 depending on whether $r$ is odd or even. For all $k \in\{1,2, \ldots,(p-1)\}$ and $s_{k}=\left(k+\left(\delta_{k}-1\right) p\right)$, let $\nabla_{k}$ and $\kappa_{k}$ be the matrices $\left(\begin{array}{cc}1 & 1 \\ s_{k} p & s_{k} p+2\end{array}\right)$ and $\left(\begin{array}{cc}1+s_{k} p & s_{k} p+3 \\ 2 s_{k} p & 2\left(s_{k} p+2\right)\end{array}\right)$.

For $\alpha=\left(\begin{array}{ll}a & b \\ c & d\end{array}\right) \in \mathrm{GL}_{2}(\mathbb{Q})$ with $\operatorname{det}(\alpha)>0$ and a weight 2 modular form $f$, define the weight 2 operator $[\alpha]$ on $f$ by $f[\alpha]=\operatorname{det}(\alpha)(c z+d)^{-2} f(\alpha(z))$. The function $f[\alpha]$ is again a modular form of weight 2 . Let $L(f, 1)$ be the special value at $s=1$ of the $L$-function $L(f, s)$ attached to a weight 2 modular form $f$. By [11, p. 48], $L(f, 1)$ is determined by the value of the Mellin transform of $f$ at $s=1$. Since the Mellin transformation of $f$ can be analytically continued to a meromorphic function on the complex plane with possible simple poles only at 0 and $2, L(f, 1)$ is finite.

Define the function $F_{E}: \mathbb{P}^{1}\left(\mathbb{Z} / p^{2} \mathbb{Z}\right) \rightarrow K$ as follows:

$$
F_{E}(x)= \begin{cases}\frac{1}{2 \pi i}\left(2 L\left(E\left[\alpha_{r}\right], 1\right)-L\left(E\left[\beta_{r}\right], 1\right)\right) & \text { if } x=(r-1, r+1), \\ \int_{0}^{\infty} 2\left(2 E\left[\nabla_{k}\right]-E\left[\kappa_{k}\right]\right) d z & \text { if } x=(1+k p, 1), \\ -F_{E}((k p+1,1)) & \text { if } x=(k p-1,1), \\ 0 & \text { if } x=( \pm 1,1) .\end{cases}
$$

We can write $L\left(E\left[\alpha_{r}\right], 1\right)$ and $L\left(E\left[\beta_{r}\right], 1\right)$ explicitly in terms of the Dedekind symbols associated to $E$ as in p. 71 of [11. We now state the main theorem of this paper.

Theorem 1.1. Let $E \in E_{2}\left(\Gamma_{0}\left(p^{2}\right)\right)$ be an Eisenstein series such that the Fourier coefficients at $\infty$ of $E$ are in a fixed number field $K$. If $E$ is a well-known basis element of $E_{2}\left(\Gamma_{0}\left(p^{2}\right)\right)$ (Lemma 3.1), then

$$
\mathcal{E}=\sum_{g \in \mathbb{P}^{1}\left(\mathbb{Z} / p^{2} \mathbb{Z}\right)} F_{E}(g) \zeta(g)
$$

In general, there is an explicit function $G_{E}: \mathbb{P}^{1}\left(\mathbb{Z} / p^{2} \mathbb{Z}\right) \rightarrow K$ such that $\mathcal{E}=$ $\sum_{g \in \mathbb{P}^{1}\left(\mathbb{Z} / p^{2} \mathbb{Z}\right)} G_{E}(g) \zeta(g)$.

In 10, Merel used the explicit expression of the Eisenstein element and the winding element for $N=p$ to prove the uniform boundedness conjecture for torsion points on elliptic curves over number fields.

We end this article by explicitly writing the winding element for the congruence subgroup $\Gamma_{0}\left(p^{2}\right)$. The winding element is interesting from the point of view of the Birch and Swinnerton-Dyer conjecture [1]. We also hope that the explicit expression of the winding element will help us to understand the Eisenstein ideals of the Hecke algebras for the general level.

Remark 1.2. Let $\alpha, \beta, s, \alpha_{j}, \beta_{k}, \sigma$ and $h$ be the matrices $\left(\begin{array}{ll}1 & 1 \\ 0 & 1\end{array}\right),\left(\begin{array}{ll}1 & 2 \\ 0 & 1\end{array}\right)$, $\left(\begin{array}{cc}0 & -1 \\ 1 & 0\end{array}\right),\left(\begin{array}{cc}0 & -1 \\ 1 & j\end{array}\right),\left(\begin{array}{cc}1 & 0 \\ k & 1\end{array}\right),\left(\begin{array}{cc}1-p^{2} & -p^{2} \\ p^{2} & 1+p^{2}\end{array}\right)$ and $\left(\begin{array}{ll}1 & 1 \\ 0 & 2\end{array}\right)$ respectively. 


\section{INTERSECTION WITH $\Gamma(2)$}

By tensoring the usual short exact sequence with $K$, we have a short exact sequence:

$$
0 \rightarrow \mathrm{H}_{1}\left(X_{0}(N), K\right) \rightarrow \mathrm{H}_{1}\left(X_{0}(N), \text { cusps, } K\right) \rightarrow K^{\text {cusps }} \rightarrow K \rightarrow 0 .
$$

The first map is a canonical injection. The second map (boundary map) $\delta$ takes a geodesic, joining the cusps $r$ and $s$ to the formal symbol $[r]-[s]$, and the third map is the sum of the coefficients. There is a canonical bijection $\Gamma_{0}\left(p^{2}\right) \backslash \mathrm{SL}_{2}(\mathbb{Z}) \cong$ $\mathbb{P}^{1}\left(\mathbb{Z} / p^{2} \mathbb{Z}\right)$ given by $\left(\begin{array}{ll}a & b \\ c & d\end{array}\right) \rightarrow(c, d)$.

Lemma 2.1. The set $T=\left\{I, \alpha_{t}, \beta_{k p} \mid 0 \leq t \leq p^{2}-1,1 \leq k \leq(p-1)\right\}$ forms a complete set of coset representatives of $\mathbb{P}^{1}\left(\mathbb{Z} / p^{2} \mathbb{Z}\right)$.

Proof. The orbits $\Gamma_{0}\left(p^{2}\right) \alpha_{t}$ and $\Gamma_{0}\left(p^{2}\right) \beta_{k}$ are disjoint since the matrices $\alpha_{t} \alpha_{t^{\prime}}^{-1}$, $\beta_{k p} \beta_{k^{\prime} p}^{-1}$ and $\alpha_{t} \beta_{k p}^{-1}$ do not belong to $\Gamma_{0}\left(p^{2}\right)$. There are $p^{2}+p-1+1=p^{2}+p=$ $\left|\mathbb{P}^{1}\left(\mathbb{Z} / p^{2} \mathbb{Z}\right)\right|$ coset representatives.

Lemma 2.2. The cusps of $\Gamma_{0}\left(p^{2}\right)$ can be identified with the set

$$
\left\{0, \infty, \frac{1}{p}, \ldots, \frac{1}{(p-1) p}\right\} .
$$

Proof. Let $P=\left\{\left(\begin{array}{cc}1 & n \\ 0 & 1\end{array}\right), n \in \mathbb{Z}\right\}$ be the parabolic subgroup inside the modular group $\mathrm{SL}_{2}(\mathbb{Z})$. There is a well-known bijection between the set of cusps $\Gamma_{0}\left(p^{2}\right) \backslash \mathbb{P}^{1}(\mathbb{Q})$ and the double coset space $\Gamma_{0}\left(p^{2}\right) \backslash \mathrm{SL}_{2}(\mathbb{Z}) / P$. The statement is obvious from the previous lemma and this bijection.

Let $\Gamma(2)$ be the subgroup of $\mathrm{SL}_{2}(\mathbb{Z})$ consisting of all matrices which are identity modulo the reduction map mod 2 and let $\Gamma=\Gamma_{0}\left(p^{2}\right) \cap \Gamma(2)$. We consider the corresponding modular curve $X_{\Gamma}=\Gamma \backslash \mathbb{H} \cup \mathbb{P}^{1}(\mathbb{Q})$. Let $\pi_{\Gamma}: \mathbb{H} \cup \mathbb{P}^{1}(\mathbb{Q}) \rightarrow X_{\Gamma}$ be the canonical surjection. Let us consider the matrices $\gamma_{k}=\left(\begin{array}{cc}\left(s_{k} p\right)^{3} & -1+s_{k}^{2} p^{2} \\ 1+s_{k}^{2} p^{2} & p s_{k}\end{array}\right)$ and

$$
\alpha_{\infty}=\left(\begin{array}{cc}
p^{2} & -1+p^{2} \\
1+p^{2} & p^{2}
\end{array}\right)
$$

Lemma 2.3. The set

$$
S=\left\{I, \gamma_{k}, \beta_{t+\delta_{t} p^{2}} \mid 0 \leq t \leq\left(p^{2}-1\right), 1 \leq k \leq(p-1)\right\} \subset \Gamma(2)
$$

forms an explicit set of coset representatives of $\mathbb{P}^{1}\left(\mathbb{Z} / p^{2} \mathbb{Z}\right)$.

Proof. An easy check shows that the orbits $\Gamma_{0}\left(p^{2}\right) \beta_{t+\delta_{t} p^{2}}, \Gamma_{0}\left(p^{2}\right) \gamma_{k}$ and $\Gamma_{0}\left(p^{2}\right) \alpha_{\infty}$ are disjoint. Since $\left|\mathbb{P}^{1}\left(\mathbb{Z} / p^{2} \mathbb{Z}\right)\right|=p^{2}+p$, the result follows.

Lemma 2.4. $\Gamma \backslash \Gamma(2)$ is isomorphic to $\mathbb{P}^{1}\left(\mathbb{Z} / p^{2} \mathbb{Z}\right)$.

Proof. There is a canonical map $s: \Gamma \backslash \Gamma(2) \rightarrow \mathbb{P}^{1}\left(\mathbb{Z} / p^{2} \mathbb{Z}\right)$ which takes a matrix to its second row. Obviously, the map is well defined and injective. The map is surjective by the previous lemma. 
Let $\pi_{0}: \Gamma \backslash \mathbb{H} \cup \mathbb{P}^{1}(\mathbb{Q}) \rightarrow \Gamma(2) \backslash \mathbb{H} \cup \mathbb{P}^{1}(\mathbb{Q})$ be the map $\pi_{0}(\Gamma z)=\Gamma(2) z$. Now $\Gamma(2) \backslash \mathbb{H} \cup \mathbb{P}^{1}(\mathbb{Q})$ contains three cusps $\Gamma(2) 1, \Gamma(2) 0, \Gamma(2) \infty$. Let $P_{-}$be the inverse image of $\Gamma(2) 1$ under the map $\pi_{0}$ and let $P_{+}$be the inverse image of $\Gamma(2) 0$ and $\Gamma(2) \infty$ under the map $\pi_{0}$. We follow [9] and study the relative homology groups $\mathrm{H}_{1}\left(X_{\Gamma}-P_{-}, P_{+}, \mathbb{Z}\right)$ and $\mathrm{H}_{1}\left(X_{\Gamma}-P_{+}, P_{-}, \mathbb{Z}\right)$. The intersection pairing is a nondegenerate bilinear pairing $\mathrm{H}_{1}\left(X_{\Gamma}-P_{+}, P_{-}, \mathbb{Z}\right) \times \mathrm{H}_{1}\left(X_{\Gamma}-P_{-}, P_{+}, \mathbb{Z}\right) \rightarrow \mathbb{Z}$. Recall the following two fundamental theorems from $\left[9\right.$. For $g \in \Gamma \backslash \Gamma(2)$, let $[g]^{0}$ (respectively $\left.[g]_{0}\right)$ be the image in $X_{\Gamma}$ of the geodesic in $\mathbb{H} \cup \mathbb{P}^{1}(\mathbb{Q})$ joining $g 0$ and $g \infty$ (respectively $g 1$ and $g(-1)$ ). If the Fourier coefficients at $\infty$ of an Eisenstein series $E$ are in a fixed number field $K$, we tensor the isomorphism in Theorem 5 of 9 ] with $K$ and obtain the following theorem.

Theorem $2.5(9])$. Let

$$
\zeta_{0}: K^{\Gamma \backslash \Gamma(2)} \rightarrow \mathrm{H}_{1}\left(X_{\Gamma}-P_{+}, P_{-}, K\right)
$$

be the map which takes $g \in \Gamma \backslash \Gamma(2)$ to the element $[g]_{0}$ and let

$$
\zeta^{0}: K^{\Gamma \backslash \Gamma(2)} \rightarrow \mathrm{H}_{1}\left(X_{\Gamma}-P_{-}, P_{+}, K\right)
$$

be the map which takes $g \in \Gamma \backslash \Gamma(2)$ to the element $[g]^{0}$. The homomorphisms $\zeta_{0}$ and $\zeta^{0}$ are isomorphisms.

Theorem $2.6([9)$. For $g, h \in \Gamma(2)$, we have

$$
[g]_{0} \circ[h]^{0}=1
$$

if $\Gamma g=\Gamma h$ and

$$
[g]_{0} \circ[h]^{0}=0
$$

otherwise.

Lemma 2.7. For $k \in\{1,2, \ldots, p-1\}$, let $s_{k}$ be defined as in the introduction. Every element of the set $P_{-}$can be written in the form $\Gamma k$ for some $k$ in the set

$$
\left\{1, \frac{1}{p^{2}}, \frac{1}{s_{p}}, \ldots, \frac{1}{s_{(p-1) p}}\right\} .
$$

Proof. Since $P_{-}=\pi_{0}^{-1}(\Gamma(2) 1)$, we can write every element of the set $P_{-}$as $\Gamma \theta 1$ for some $\theta \in S$ (Lemma 2.3). If $\theta=\beta_{j}$ and $(j+1, p)=1$, then an easy calculation shows that $\beta_{j}(1) \in \Gamma 1$. Similarly, we see that $\alpha_{\infty}(1)$ and $\gamma_{k}(1)$ belong to $\Gamma 1$. We deduce that $\beta_{j}(1) \in P_{-}-\Gamma 1$ if and only if $(j+1, p) \neq 1$.

Let $\pi, \pi^{\prime}: \Gamma \backslash \mathbb{H} \cup \mathbb{P}^{1}(\mathbb{Q}) \rightarrow \Gamma_{0}\left(p^{2}\right) \backslash \mathbb{H} \cup \mathbb{P}^{1}(\mathbb{Q})$ be the maps $\pi(\Gamma z)=\Gamma_{0}\left(p^{2}\right) z$ and $\pi^{\prime}(\Gamma z)=\Gamma_{0}\left(p^{2}\right) \frac{z+1}{2}$ respectively.

Lemma 2.8. Let $f: X_{0}\left(p^{2}\right) \rightarrow \mathbb{C}$ be a rational function. Then $\frac{(f \circ \pi)^{2}}{f \circ \pi^{\prime}}$ has no zeros or poles in $P_{-}$.

Proof. For integers $s, t$ such that $(s, t, p)=1$, the local coordinates around the point $\Gamma_{0}\left(p^{2}\right) 0, \Gamma_{0}\left(p^{2}\right) \infty$ and $\Gamma_{0}\left(p^{2}\right) \frac{s}{t p}$ are given by $q_{0}(z)=e^{2 \pi i \frac{1}{-p^{2} z}}, q_{\infty}(z)=e^{2 \pi i z}$ and $q_{\frac{s}{t p}}(z)=e^{2 \pi i \frac{z}{s(-t p z+s)}}$ respectively (cf. [5], p. 33). In the modular curve $X_{\Gamma}$, the local coordinates around the points of $P_{-}$are given by $q_{1}(z)=e^{2 \pi i \frac{1}{2 p^{2}(-z+1)}}$, $q_{\frac{1}{p^{2}}}(z)=e^{2 \pi i \frac{z}{2\left(-p^{2} z+1\right)}}$ and $q_{\frac{1}{s_{k} p}}(z)=e^{2 \pi i \frac{z}{2\left(-s_{k} p z+1\right)}}$. 
Now around the point $\Gamma 1$ and $\Gamma \frac{1}{p^{2}}$, we have the equalities $q_{0} \circ \pi=q_{1}^{2}, q_{0} \circ \pi^{\prime}=q_{1}^{4}$ and, $q_{\frac{1}{p^{2}}} \circ \pi=q_{\frac{1}{p^{2}}}^{2}, q_{\frac{1}{p^{2}}} \circ \pi^{\prime}=q_{\frac{1}{p^{2}}}^{4}$.

We prove similar equalities around each of the points $\Gamma \frac{1}{s_{k} p}$. Let $1+s_{k} p=2 k^{\prime}$; then we have $\pi^{\prime}\left(\frac{1}{s_{k} p}\right)=\frac{k^{\prime}}{s_{k} p}$ and $\pi\left(\frac{1}{s_{k} p}\right)=\frac{1}{s_{k} p}$. The maps $\pi^{\prime}$ and $\pi$ take the points $\frac{1}{s_{k} p}$ of $P_{-}$to different cusps. Nevertheless a small calculation shows that $q_{\frac{s_{k}}{k^{\prime} p}} \circ \pi^{\prime}(z)=\left(q \frac{s_{k}}{\left(1+2 s_{k} p\right) p} \circ \pi\right)^{2}(z)$. Since $\Gamma_{0}\left(p^{2}\right) \frac{1}{k p}=\Gamma_{0}\left(p^{2}\right) \frac{1}{s_{k} p}=\Gamma_{0}\left(p^{2}\right) \frac{s_{k}}{p}=$ $\Gamma_{0}\left(p^{2}\right) \frac{s_{k}}{\left(1+2 s_{k} p\right) p}$, we obtain the lemma.

\section{EISENSTEIn SERIES FOR $\Gamma_{0}\left(p^{2}\right)$}

Corresponding to each non-trivial Dirichlet character $\phi:(\mathbb{Z} / p \mathbb{Z})^{*} \rightarrow \mathbb{C}^{*}$, we have the Eisenstein series $E_{2}^{\phi, \bar{\phi}}(\tau)=\sum_{n} \sigma_{1}^{\phi, \bar{\phi}}(n) q^{n}$, where the generalized power sum in the Fourier coefficient is $\sigma_{1}^{\phi, \bar{\phi}}(n)=\sum_{m \mid n} \phi\left(\frac{n}{m}\right) \phi(m) m$. Consider the series $E_{2}^{\prime}(\tau)=\frac{-1}{24}+\sum_{n} \sigma_{1}(n) q^{n}$, where $\sigma_{1}(n)$ denotes the sum of the positive divisors of $n$. Let $E_{1}$ and $E_{2}$ be the Eisenstein series

$$
\begin{gathered}
E_{1}(\tau)=E_{2}^{\prime}(\tau)-p E_{2}^{\prime}(p \tau), \\
E_{2}(\tau)=E_{2}^{\prime}(\tau)-p^{2} E_{2}^{\prime}\left(p^{2} \tau\right)
\end{gathered}
$$

respectively.

Lemma 3.1. The set $\mathbb{E}=\left\{E_{1}, E_{2}, E_{2}^{\phi, \bar{\phi}}\right\}$ represents a basis of $E_{2}\left(\Gamma_{0}\left(p^{2}\right)\right)$.

Proof. Let $N=p^{2}$ in [3, Theorem 4.6.2].

By [11, p. 36], the map $\delta: E_{2}\left(\Gamma_{0}(N)\right) \longrightarrow \operatorname{Div}^{0}$ (cusps) is an isomorphism. For all cusps $x$, let $e_{\Gamma_{0}\left(p^{2}\right)}(x)$ denote the ramification index of $x$ over $\mathrm{SL}_{2}(\mathbb{Z}) \backslash\left(\mathbb{H} \cup \mathbb{P}^{1}(\mathbb{Q})\right)$. The Eisenstein series $E$ corresponds to the divisor

$$
\delta(E)=\sum_{x \in \operatorname{Cusps}\left\{\Gamma_{0}\left(p^{2}\right)\right\}} e_{\Gamma_{0}\left(p^{2}\right)}(x) a_{0}(E[x])\{x\} .
$$

The congruence subgroup $\Gamma_{0}\left(p^{2}\right)$ has $(p+1)$ cusps as listed in Lemma 2.2, By [12, p. 538], we see that

$$
e_{\Gamma_{0}\left(p^{2}\right)}(x)= \begin{cases}1 & \text { if } x=\frac{1}{k p} \\ 1 & \text { if } x=\frac{1}{p^{2}} \\ p^{2} & \text { if } x=0\end{cases}
$$

3.1. Period homomorphisms of the Eisenstein series. For any $z_{0} \in \mathbb{H}$ and $\gamma \in \Gamma_{0}\left(p^{2}\right)$, let $c(\gamma)$ be the class in $\mathrm{H}_{1}\left(Y_{0}\left(p^{2}\right), \mathbb{Z}\right)$ of the image in $Y_{0}\left(p^{2}\right)$ of the geodesic in $\mathbb{H}$ joining $z_{0}$ and $\gamma\left(z_{0}\right)$. This class is independent of the choice of $z_{0} \in \mathbb{H}$. Suppose the Fourier coefficients at $\infty$ of an Eisenstein series $E \in E_{2}\left(\Gamma_{0}\left(p^{2}\right)\right)$ are in a fixed number field $K$. The period of the Eisenstein series $E$ is the map $\pi_{E}: \mathrm{H}_{1}\left(Y_{0}\left(p^{2}\right), K\right) \rightarrow K$ [11, p. 43]:

$$
\pi_{E}(\gamma)=\int_{c(\gamma)} E(z) d z
$$

This is a period of the holomorphic differential form $E(z) d z$ on the modular curve $Y_{0}\left(p^{2}\right)$. For integers $u$ and $v$, let $S(u, v)$ be the Dedekind sum. The following 
proposition summarizes some well-known properties of the map $\pi_{E}$. The proofs are given in [11, Prop. 2.3.3] and [8, p. 216].

Proposition 3.2. Let $\gamma=\left(\begin{array}{ll}a & b \\ c & d\end{array}\right)$ be an element of $\Gamma_{0}\left(p^{2}\right)$.

(1) $\pi_{E}$ is a group homomorphism.

(2) $\pi_{E}(\gamma)=\frac{a+d}{c} a_{0}(E)-\frac{1}{2 \pi i} L\left(E\left[\left(\begin{array}{cc}1 & -d \\ 0 & c\end{array}\right)\right], 1\right)$ if $c \neq 0$ and $\pi_{E}(\gamma)=\frac{b}{d} a_{0}(E)$ if $c=0$.

(3) For the Eisenstein series $E_{2}$, we have

$$
\frac{1}{\pi i} L\left(E_{2}\left[\left(\begin{array}{cc}
1 & -d \\
0 & c
\end{array}\right)\right], 1\right)=-\operatorname{sgn}(c)\left(S(d,|c|)-S\left(d, \frac{|c|}{p^{2}}\right)\right),
$$

where $\operatorname{sgn}(c)=\frac{c}{|c|}$ denotes the sign of $c$.

\section{EISENSTEIN ELEMENT}

For $E \in E_{2}\left(\Gamma_{0}\left(p^{2}\right)\right)$, let $\lambda_{E}: X_{0}\left(p^{2}\right) \rightarrow \mathbb{C}$ be the rational function whose logarithmic derivative is $2 \pi i E(z) d z=2 \pi i \omega_{E}$. Consider the rational function $\lambda_{E, 2}=\frac{\left(\lambda_{E} \circ \pi\right)^{2}}{\lambda_{E} \circ \pi^{\prime}}$ on $X_{\Gamma}$. By Lemma 2.8, it has no zeros and poles in $P_{-}$. Let $k^{*}\left(\omega_{E}\right)$ be the logarithmic derivative of the function. Let $\psi_{E}(c)=\int_{c} k^{*}\left(\omega_{E}\right)$ be the corresponding "period" homomorphism $\mathrm{H}_{1}\left(X_{\Gamma}-P_{+}, P_{-}, K\right) \rightarrow K$.

By the non-degeneracy of the intersection pairing, there is a unique element $\mathcal{E}_{0} \in \mathrm{H}_{1}\left(X_{\Gamma}-P_{-}, P_{+}, K\right)$ such that $\mathcal{E}_{0} \circ c=\psi_{E}(c)$ for all $c \in \mathrm{H}_{1}\left(X_{\Gamma}-P_{+}, P_{-}, K\right)$. By Theorem 2.5, we write $\mathcal{E}_{0}$ uniquely as $\mathcal{E}_{0}=\sum_{g \in \mathbb{P}^{1}\left(\mathbb{Z} / p^{2} \mathbb{Z}\right)} F_{0}(g) \zeta^{0}(g)$. Let $F_{E}$ : $\mathbb{P}^{1}\left(\mathbb{Z} / p^{2} \mathbb{Z}\right) \rightarrow K$ be the function as defined in the introduction.

Lemma 4.1. For all $g \in \mathbb{P}^{1}\left(\mathbb{Z} / p^{2} \mathbb{Z}\right)$ and for all $E \in E_{2}\left(\Gamma_{0}\left(p^{2}\right)\right)$, we have

$$
\psi_{E}\left(\zeta_{0}(g)\right)=F_{E}(g) .
$$

Proof. Since every Eisenstein series is a linear combination of the basis element and the intersection product $\circ$ is bilinear, it is enough to prove the statement for the basis of $E_{2}\left(\Gamma_{0}\left(p^{2}\right)\right)$ as described in Lemma 3.1. If $x$ is not of the form $(l p \pm 1,1)$ or $(1, l p \pm 1)$, then we write $x$ as $(r-1, r+1)$ and choose $s$ such that $r s \equiv 1 \bmod 4 p^{2}$. We consider the matrix $V(r, s)=\left(\begin{array}{cc}\frac{r-3}{2} & \frac{r-1}{2} \\ \frac{1-r}{2} & \frac{-1-r}{2}\end{array}\right)\left(\begin{array}{ll}1 & 2 \\ 0 & 1\end{array}\right)\left(\begin{array}{cc}\frac{s-3}{2} & \frac{s-1}{2} \\ \frac{1-s}{2} & \frac{-1-s}{2}\end{array}\right)$.

By a calculation similar to Proposition 7 of 9 , we have

$$
\psi_{E}\left(\zeta_{0}(x)\right)=-2 \pi_{E}(V(r, s))+\pi_{E}\left(h V(r, s) h^{-1}\right),
$$

and hence $\psi_{E}\left(\zeta_{0}(x)\right)=F_{E}(x)$. We now prove that

$$
\psi_{E}\left(\zeta_{0}(k p+1,1)\right)=F_{E}((k p+1,1)) .
$$

Consider the function $f(z)=2 E(z)-\frac{1}{2} E\left(\frac{z+1}{2}\right)$. Clearly, $f$ is a modular form of weight two with respect to the congruence subgroup $\Gamma$. We have

$$
\int_{\beta_{1+s_{k} p} 1}^{\beta_{1+s_{k} p}(-1)} f(z) d z=\int_{\frac{1}{s_{k} p+2}}^{\frac{1}{s_{k} p}} f(z) d z=\int_{0}^{\infty} 2 f\left[\nabla_{k}\right](z) d z=F_{E}((k p+1,1)) .
$$


By replacing $g_{1}$ in [9] by $\beta_{1+s_{k} p}$, we see that

$$
\begin{aligned}
F_{E}((1+k p, 1)) & =\psi_{E}\left(\zeta_{0}\left(\beta_{1+s_{k} p}\right)\right) \\
& =-\int_{\beta_{-1+s_{k} p}(1)}^{\beta_{-1+s_{k} p}(-1)} f(\sigma z) d(\sigma z)=-\psi_{E}\left(\zeta_{0}\left(\beta_{-1+s_{k} p}\right)\right) .
\end{aligned}
$$

By a similar substitution of $g_{1}$ in $[9]$ by $\beta_{1+p^{2}}$, we see that $\psi_{E}\left(\zeta_{0}\left(\left(1+p^{2}, 1\right)\right)\right)=$ $-\psi_{E}\left(\zeta_{0}\left(\left(-1+p^{2}, 1\right)\right)\right)$. If $E$ is an Eisenstein series with real Fourier coefficients, then we have $\psi_{E}\left(\zeta_{0}(( \pm 1,1))\right)=F_{E}(( \pm 1,1))=0$. For $E_{2}^{\phi, \bar{\phi}}$, we observe that $E_{2}^{\phi, \bar{\phi}}+E_{2}^{\bar{\phi}, \phi}$ and $i\left(E_{2}^{\phi, \bar{\phi}}-E_{2}^{\bar{\phi}, \phi}\right)$ are Eisenstein series with real Fourier coefficients. Similarly, we obtain $\psi_{E_{2}^{\phi, \bar{\phi}}}\left(\zeta_{0}(( \pm 1,1))\right)=F_{E_{2}^{\phi, \bar{\phi}}}(( \pm 1,1))=0$.

Proposition 4.2. For all $E \in E_{2}\left(\Gamma_{0}\left(p^{2}\right)\right)$, let us consider the element $\mathcal{E}_{0}$ of $\mathrm{H}_{1}\left(X_{\Gamma}-P_{+}, P_{-}, K\right)$ defined by $\mathcal{E}_{0}=\sum_{g \in \mathbb{P}^{1}\left(\mathbb{Z} / p^{2} \mathbb{Z}\right)} F_{E}(g) \zeta^{0}(g)$. For all

$$
c \in \mathrm{H}_{1}\left(X_{\Gamma}-P_{+}, P_{-}, K\right),
$$

we have $\mathcal{E}_{0} \circ c=\psi_{E}(c)$.

Proof. This is obvious from Lemma 4.1 and Theorem 2.6.

Corresponding to each non-trivial character $\phi:(\mathbb{Z} / p \mathbb{Z})^{*} \rightarrow \mathbb{C}^{*}$, define

$$
t_{\phi}=\sum_{d=0}^{p-1} \sum_{e=0}^{p-1} \phi(d)^{2} \sum_{l \equiv(d+e p)}^{\prime} \frac{1}{\left(\bmod p^{2}\right)} \bar{l}^{2} .
$$

Lemma 4.3. The constant Fourier coefficients of $E_{2}^{\phi, \bar{\phi}}$ at the cusps $\frac{1}{k p}$ are constant multiples of $\phi(k) t_{\phi}$.

Proof. Let $\zeta_{+}^{n}(k)=\sum_{m=1, m \equiv n}^{\infty}\left(\bmod p^{2}\right) \frac{1}{m^{k}}$ be the modified zeta function. For $\bar{v} \in\left(\mathbb{Z} / p^{2} \mathbb{Z}\right)^{2}$, consider the series $E_{2}^{\bar{v}}(z)=\sum_{(c, d) \equiv \bar{v}\left(\bmod p^{2}\right)} \frac{1}{(c z+d)^{2}}$ and $G_{2}^{\bar{v}}=$ $\sum_{n \in\left(\mathbb{Z} / p^{2} \mathbb{Z}\right)^{*}} \zeta_{+}^{n}(2) E_{2}^{n^{-1} \bar{v}}$. Let $G_{2}^{\phi, \bar{\phi}}=\sum_{c=0}^{p-1} \sum_{d=0}^{p-1} \sum_{e=0}^{p-1} \phi(c) \phi(d) G_{2}^{\overline{(c p, d+e p)}}(\tau)$ be the Eisenstein series for the congruence subgroup $\Gamma_{0}\left(p^{2}\right)$. We show that the constant Fourier coefficients of the Eisenstein series $G_{2}^{\phi, \bar{\phi}}$ at the cusps $\frac{1}{k p}$ are constant multiples of $\phi(k) t_{\phi}$. Since $\beta_{k p} \in \mathrm{SL}_{2}(\mathbb{Z})$ is such that $\frac{1}{k p}=\beta_{k p}(\infty)$, the behavior of $G_{2}^{\phi, \bar{\phi}}$ at the cusp $\frac{1}{k p}$ is described by the Fourier series expansion of $G_{2}^{\phi, \bar{\phi}}\left[\beta_{k p}\right]$.

A small check [3, Prop. 4.2.1] shows that for all $\gamma \in \mathrm{SL}_{2}(\mathbb{Z}), E_{2}^{\bar{v}}[\gamma](z)=E_{2}^{\overline{v \gamma}}(z)$. Since $G_{2}^{\bar{v}}$ is a linear combination of $E_{2}^{\bar{v}}$, we have

$$
G_{2}^{\phi, \bar{\phi}}\left[\beta_{k p}\right]=\sum_{c=0}^{p-1} \sum_{d=0}^{p-1} \sum_{e=0}^{p-1} \phi(c) \phi(d) G_{2}^{\overline{(c p, d+e p) \beta_{k p}}}(\tau) .
$$

For $(c p, d+e p) \in\left(\mathbb{Z} / p^{2} \mathbb{Z}\right)^{2}$, we have an equality

$$
(c p, d+e p) \beta_{k p}=(c p+k p(d+e p), d+e p) .
$$

If $\left(c_{v}, d_{v}\right)=\bar{v} \in\left(\mathbb{Z} / p^{2} \mathbb{Z}\right)^{2}$, then the constant terms of $G_{2}^{\bar{v}}$ are non-zero if and only if $c_{v} \equiv 0\left(\bmod p^{2}\right)$. So, the constant Fourier coefficients of $G_{2}^{\phi, \bar{\phi}}$ at the cusps $\frac{1}{k p}$ are $\phi(-k) t_{\phi}$. Since $E_{2}^{\phi, \bar{\phi}}$ is a constant multiple of $G_{2}^{\phi, \bar{\phi}}$, the result follows. 
Lemma 4.4. The constant Fourier coefficients of $E_{1}$ and $E_{2}$ at the cusps $\frac{1}{k p}$ are $\frac{p-1}{24}$ and 0 respectively.

Proof. We first prove that the constant coefficients for the Fourier expansions of $E_{2}$ at the cusps $\frac{1}{k p}$ are 0 . As usual, the constant terms of the Fourier expansions of $E_{2}$ at the cusps $\frac{1}{k p}$ are the constant terms at $\infty$ of $E_{2}\left[\beta_{k p}\right]$.

Let $\Delta$ be the standard Ramanujan cusp form of weight 12. By [5, p. 28], we have $\frac{d}{d z} \log \Delta\left(\beta_{k p}(z)\right)=12 \frac{d}{d z} \log (k p z+1)+\frac{d}{d z} \log \Delta(z)$. If $m$ and $l$ are integers such that $m p-k l=1$, then

$$
\begin{gathered}
\Delta\left(\frac{p^{2} z}{k p z+1}\right)=\Delta\left(\left(\begin{array}{cc}
p & 0 \\
k & 1
\end{array}\right) p z\right)=\Delta\left(\left(\begin{array}{cc}
p & l \\
k & m
\end{array}\right)\left(\begin{array}{cc}
1 & -l \\
0 & p
\end{array}\right) p z\right) \\
=\Delta\left(\left(\begin{array}{cc}
p & l \\
k & m
\end{array}\right)\left(z-\frac{l}{p}\right)\right) .
\end{gathered}
$$

By taking the logarithmic derivative, we deduce that

$$
\frac{d}{d z} \log \Delta\left(\left(\begin{array}{cc}
p & l \\
k & m
\end{array}\right)\left(z-\frac{l}{p}\right)\right)=12 \frac{d}{d z} \log (k p z+1)+\frac{d}{d z} \log \Delta\left(z-\frac{l}{p}\right) .
$$

Since $E_{2}=\frac{1}{2 \pi i} \frac{d}{d z} \log \frac{\Delta\left(p^{2} z\right)}{\Delta(z)}$, the above calculation shows that the constant terms of $E_{2}\left[\beta_{k p}\right]$ at $\infty$ are 0 . The other Eisenstein series $E_{1} \in E_{2}\left(\Gamma_{0}(p)\right)$ and each of the cusps $\frac{1}{k p}$ represents the cusp $\infty$. Hence, we deduce that $a_{0}\left(E_{1}\left[\beta_{k p}\right]\right)=\frac{p-1}{24}$ for all cusps $\frac{1}{k p}$.

Let $\pi_{*}: \mathrm{H}_{1}\left(X_{\Gamma}-P_{-}, P_{+}, \mathbb{Z}\right) \rightarrow \mathrm{H}_{1}\left(X_{0}\left(p^{2}\right)\right.$, cusp, $\left.\mathbb{Z}\right)$ be the map induced from $\pi$ by functoriality.

Proposition 4.5. For all $E \in \mathbb{E}$, let $\mathcal{E}_{0} \in \mathrm{H}_{1}\left(X_{\Gamma}-P_{-}, P_{+}, K\right)$ be the Eisenstein element as defined in Proposition 4.2. The boundary of the modular symbol $\pi_{*}\left(\mathcal{E}_{0}\right)$ is a constant multiple of $\delta(E)$.

Proof. Since $\pi_{*}\left(\zeta^{0}(g)\right)=\zeta(g)$, we explicitly write the element

$$
\pi_{*}\left(\mathcal{E}_{0}\right) \in \mathrm{H}_{1}\left(X_{0}\left(p^{2}\right) \text {, cusps, } K\right)
$$

as

$$
\pi_{*}\left(\mathcal{E}_{0}\right)=\sum_{g \in \mathbb{P}^{1}\left(\mathbb{Z} / p^{2} \mathbb{Z}\right)} F_{E}(g) \zeta(g) .
$$

Computing the boundary, using the coset representatives as in Lemma 2.1, we obtain

$$
\begin{aligned}
\delta\left(\pi_{*}\left(\mathcal{E}_{0}\right)\right) & =\sum_{k=1}^{p-1}\left[F_{E}(k p, 1)\left(\left\{\frac{1}{k p}\right\}-\{0\}\right)+F_{E}(1, k p)\left(\{0\}-\left\{\frac{1}{-k p}\right\}\right)\right] \\
& +F_{E}(0,1)(\{\infty\}-\{0\})+F_{E}(1,0)(\{0\}-\{\infty\}) .
\end{aligned}
$$

We calculate each of the terms $F_{E}(k p, 1), F_{E}(1, k p), F_{E}(0,1)$ and $F_{E}(1,0)$. For $g$ of the form $(k p, 1)$ or $(1,-k p)$, we choose $r$ and $s$ as follows:

\begin{tabular}{|c|c|c|}
\hline$g$ & $r$ & $s$ \\
\hline$(k p, 1)$ & $1+2 k p$ & $1-2 k p$ \\
\hline$(1,-k p)$ & $-1+2 k p$ & $-1-2 k p$ \\
\hline
\end{tabular}


Now $\pi_{E}(V(2 k p-1,-1-2 k p))=-\pi_{E}(V(1+2 k p, 1-2 k p))$ and

$$
\pi_{E}\left(h V(2 k p-1,-1-2 k p) h^{-1}\right)=-\pi_{E}\left(h V(1+2 k p, 1-2 k p) h^{-1}\right),
$$

so we have $F_{E}(1,-k p)=-F_{E}(k p, 1)$.

By [3. p. 166], $E\left[\beta_{k p}\right]$ is a modular form with respect to the congruence subgroup $\beta_{k p}^{-1} \Gamma_{0}\left(p^{2}\right) \beta_{k p}$ and $\alpha$ belongs to this congruence subgroup. From [11, p. 52] we see that $a_{0}\left(E\left[\beta_{k p}\right]\right)=\int_{z_{0}}^{\alpha z_{0}} E\left[\beta_{k p}\right](z) d z$ and $2 a_{0}\left(E\left[\beta_{k p}\right]\right)=\int_{z_{0}}^{\beta z_{0}} E\left[\beta_{k p}\right](z) d z$. A small computation shows that $2 a_{0}\left(E\left[\beta_{k p}\right]\right)=\pi_{E}(V(1-2 k p, 1+2 k p))$ and $a_{0}\left(E\left[\beta_{2 k p}\right]\right)=$ $\pi_{E}\left(h V(1+2 k p, 1-2 k p) h^{-1}\right)$ so that $F_{E}(k p, 1)=-4 a_{0}\left(E\left[\beta_{k p}\right]\right)+a_{0}\left(E\left[\beta_{2 k p}\right]\right)$.

Similarly, we obtain $F_{E}((0,1))=-3 a_{0}(E)$ and $F_{E}((1,0))=3 a_{0}(E)$. Hence, the boundary of $\pi_{*}\left(\mathcal{E}_{0}\right)$ is

$$
\begin{gathered}
-\sum_{k=1}^{p-1} 2\left(4 a_{0}\left(E\left[\frac{1}{k p}\right]\right)-a_{0}\left(E\left[\frac{1}{2 k p}\right]\right)\right)\left\{\frac{1}{k p}\right\} \\
-6 a_{0}(E)\{\infty\}+\left[\sum_{k=1}^{p-1} 2\left(4 a_{0}\left(E\left[\frac{1}{k p}\right]\right)-a_{0}\left(E\left[\frac{1}{2 k p}\right]\right)\right)+6 a_{0}(E)\right]\{0\} .
\end{gathered}
$$

Suppose the Eisenstein form is $E=E_{2}^{\phi, \bar{\phi}}$; we conclude that

$$
\delta\left(\pi_{*}\left(\mathcal{E}_{0}\right)\right)=(2 \phi(2)-8) \delta(E)
$$

(Lemma 4.3). If we now consider the Eisenstein series $E=E_{1}$ or $E=E_{2}$, we see the boundary of $\pi_{*}\left(\mathcal{E}_{0}\right)$ is $-6 \delta(E)$ (Lemma 4.4).

Remark 4.6. We note that for the congruence subgroup $\Gamma_{0}(N)$, we may calculate the boundary in a similar manner. For the congruence subgroup $\Gamma_{0}\left(p^{3}\right)$ and $(p, 1) \in \mathbb{P}^{1}\left(\mathbb{Z} / p^{3} \mathbb{Z}\right)$, a suitable choice for $r$ and $s$ is $1+2\left(p^{2} \pm p\right)$ respectively. Unfortunately, we cannot relate $\pi_{E}(V(r, s))$ and $\pi_{E}\left(h V(r, s) h^{-1}\right)$ with Fourier coefficients of the Eisenstein series. We also observe that for distinct odd primes $p, q$ with $p \equiv$ $\pm 1(\bmod q),(p, 1) \in \mathbb{P}^{1}(\mathbb{Z} / p q \mathbb{Z})$ cannot be written as $(r-1, r+1)$.

Proposition 4.7. For all $E \in E_{2}\left(\Gamma_{0}\left(p^{2}\right)\right)$, let $\mathcal{E} \in \mathrm{H}_{1}\left(X_{0}\left(p^{2}\right)\right.$, cusps, $\left.K\right)$ be the corresponding Eisenstein element. The boundary of $\mathcal{E}$ is $-\delta(E)$.

Proof. Since the map $\zeta: \mathrm{SL}_{2}(\mathbb{Z}) \rightarrow \mathrm{H}_{1}\left(X_{0}\left(p^{2}\right)\right.$, cusps, $\left.\mathbb{Z}\right)$ is surjective, we write

$$
\mathcal{E}=\sum_{g \in \mathbb{P}^{1}\left(\mathbb{Z} / p^{2} \mathbb{Z}\right)} G_{E}(g) \zeta(g) .
$$

We again use the coset representatives as in Lemma 2.1 then the boundary of $\mathcal{E}$ is

$$
\begin{aligned}
& \sum_{k=1}^{p-1}\left[G_{E}(k p, 1)\left(\left\{\frac{1}{k p}\right\}-\{0\}\right)+G_{E}(1, k p)\left(\{0\}-\left\{\frac{1}{-k p}\right\}\right)\right] \\
& +G_{E}(0,1)(\{\infty\}-\{0\})+G_{E}(1,0)(\{0\}-\{\infty\}) .
\end{aligned}
$$

Let $\rho=\frac{1+\sqrt{-3}}{2}$ and $\rho^{*}=-\bar{\rho}$ be the points on $\mathbb{H}$. For all $g \in \mathrm{SL}_{2}(\mathbb{Z})$, let $g\left\{\rho, \rho^{*}\right\}$ be the image in $X_{0}\left(p^{2}\right)(\mathbb{C})$ of the geodesic in $\mathbb{H}$ joining the points $g . \rho$ and $g . \rho^{*}$. Since the intersection product $\circ$ is bilinear, we have

$$
\mathcal{E} \circ g\left\{\rho, \rho^{*}\right\}=\sum_{k \in \mathbb{P}^{1}\left(\mathbb{Z} / p^{2} \mathbb{Z}\right)} G_{E}(k) \zeta(k) \circ g\left\{\rho, \rho^{*}\right\} .
$$


From the consideration of the fundamental domain [13, we conclude that

$$
g\{0, \infty\} \circ h\left\{\rho, \rho^{*}\right\}= \begin{cases}1 & \text { if } \Gamma_{0}\left(p^{2}\right) g=\Gamma_{0}\left(p^{2}\right) h \\ -1 & \text { if } \Gamma_{0}\left(p^{2}\right) g=\Gamma_{0}\left(p^{2}\right) h s \\ 0 & \text { otherwise }\end{cases}
$$

As a consequence, we deduce that $\mathcal{E} \circ h\left\{\rho, \rho^{*}\right\}=\int_{h \rho}^{h \rho^{*}} E(z) d z=G_{E}(h)-G_{E}(h s)$. Let $x=\frac{1}{k p}$ be a cusp; then $\beta_{k p}$ is a matrix that takes $\infty$ to $\frac{1}{k p}$. Now $E\left[\beta_{k p}\right]$ is a modular form with respect to the congruence subgroup $\beta_{k p}^{-1} \Gamma_{0}\left(p^{2}\right) \beta_{k p}$, and $\alpha$ belongs to this congruence subgroup. By [11, p. 52], we have $a_{0}\left(E\left[\frac{1}{k p}\right]\right)=\int_{z_{0}}^{\alpha z_{0}} E\left[\beta_{k p}\right](z) d z$ so that

$$
G_{E}\left(\beta_{k p} s\right)-G_{E}\left(\beta_{k p}\right)=\int_{\beta_{k p} \rho^{*}}^{\beta_{k p} \alpha \rho^{*}} E(z) d z=a_{0}\left(E\left[\frac{1}{k p}\right]\right)=a_{0}\left(E\left[\frac{1}{k p}\right]\right) e_{\Gamma_{0}\left(p^{2}\right)}\left(\frac{1}{k p}\right) .
$$

Now consider the cusp $x=\infty$; then we have $a_{0}(E)=\int_{\rho^{*}}^{\alpha \rho^{*}} E(z) d z=\int_{\rho^{*}}^{\rho} E(z) d z$. It follows that $G_{E}(s)-G_{E}(I)=a_{0}(E) e_{\Gamma_{0}\left(p^{2}\right)}(\infty)$. Noting that

$$
\sum_{x \in \operatorname{Cusps}\left\{\Gamma_{0}\left(p^{2}\right)\right\}} e_{\Gamma_{0}\left(p^{2}\right)}(x) a_{0}(E[x])=0
$$

$\left(\delta(E) \in \operatorname{Div}^{0}(\right.$ cusps $\left.)\right)$, we obtain the proposition.

Remark 4.8. Let $\Gamma$ be any congruence subgroup. The same method may be used to calculate the boundary of the Eisenstein element corresponding to any Eisenstein series $E \in E_{2}(\Gamma)$. Let $x$ be a cusp and $g_{x} \in \mathrm{SL}_{2}(\mathbb{Z})$ take $x$ to infinity. The coefficient of $x$ in the expression of the boundary of the Eisenstein element $\mathcal{E}$ is $\int_{g_{x} \rho^{*}}^{g_{x} \rho} E(z) d z=\int_{\rho^{*}}^{\rho} E\left[g_{x}\right](z) d z$. Unfortunately, the integrals are not always easily computable. As an example, we consider the congruence subgroup $\Gamma_{0}\left(p^{3}\right)$. The rational number $\frac{1}{p}$ is a cusp with respect to this congruence subgroup but $\beta_{p} \alpha \beta_{p}^{-1}$ does not belong to $\Gamma_{0}\left(p^{3}\right)$. Nevertheless, we can calculate the integrals explicitly in terms of values of the Siegel units.

We hope to calculate the boundary map explicitly and relate it with the Fourier coefficients in our endeavor to write the explicit expression of the Eisenstein elements for general congruence subgroups.

Lemma 4.9. The integrals of every holomorphic differential over $\mathcal{E}$ and $\pi_{*}\left(\mathcal{E}_{0}\right)$ are zero.

Proof. A straightforward generalization of [9, Lemma 5].

We now prove the main theorem, Theorem 1.1, of this article.

Proof. We consider the short exact sequence of Section 2, For the Eisenstein series $E_{2}^{\phi, \bar{\phi}} \in \mathbb{E}$, the boundary of $\pi_{*}\left(\mathcal{E}_{0}\right)$ is $8-2 \phi(2)$ times the boundary of $\mathcal{E}$ (cf. Proposition 4.5). For the Eisenstein series $E_{1}$ or $E_{2} \in \mathbb{E}$, the boundary of $\pi_{*}\left(\mathcal{E}_{0}\right)$ is the same as the boundary of $6 \mathcal{E}$ (cf. Proposition 4.5)

There is a non-degenerate bilinear pairing $S_{2}\left(\Gamma_{0}\left(p^{2}\right)\right) \times \mathrm{H}_{1}\left(X_{0}\left(p^{2}\right), \mathbb{R}\right) \rightarrow \mathbb{C}$ given by $(f, c)=\int_{c} f(z) d z$. Hence, the integrals of the holomorphic differentials over 
$\mathrm{H}_{1}\left(X_{0}(N), K\right)$ are not always zero. Since the integrals of every holomorphic differential over $\mathcal{E}$ and $\pi_{*}\left(\mathcal{E}_{0}\right)$ are always zero, $\mathcal{E}$ is a constant multiple of

$$
\pi_{*}\left(\mathcal{E}_{0}\right)=\sum_{g \in \mathbb{P}^{1}\left(\mathbb{Z} / p^{2} \mathbb{Z}\right)} F_{E}(g) \zeta(g)
$$

for every $E \in \mathbb{E}$. Let $\mathcal{E}_{i}$ be the Eisenstein element corresponding to each $E_{i} \in \mathbb{E}$. For $E_{1}$ and $E_{2}$, we obtain $\mathcal{E}_{i}=\sum_{g \in \mathbb{P}^{1}\left(\mathbb{Z} / p^{2} \mathbb{Z}\right)} \frac{F_{E_{i}}(g)}{6} \zeta(g)$. For $E_{2}^{\phi, \bar{\phi}}$, we have $\mathcal{E}_{2}^{\phi, \bar{\phi}}=$ $\sum_{g \in \mathbb{P}^{1}\left(\mathbb{Z} / p^{2} \mathbb{Z}\right)} \frac{F_{E_{2}^{\phi,} \bar{\phi}}(g)}{8-2 \phi(2)} \zeta(g)$.

Let $E \in E_{2}\left(\Gamma_{0}\left(p^{2}\right)\right)$ be an arbitrary Eisenstein series; then $E=l_{1} E_{1}+l_{2} E_{2}+$ $\sum_{\phi} l_{\phi} E_{2}^{\phi, \bar{\phi}}$. Since the intersection product $\circ$ is bilinear, $\mathcal{E}=l_{1} \mathcal{E}_{1}+l_{2} \mathcal{E}_{2}+\sum_{\phi} l_{\phi} \mathcal{E}_{2}^{\phi, \bar{\phi}}$. Define the function $G_{E}: \mathbb{P}^{1}\left(\mathbb{Z} / p^{2} \mathbb{Z}\right) \rightarrow K$ by

$$
G_{E}:=\frac{l_{1} F_{E_{1}}+l_{2} F_{E_{2}}}{6}+\sum_{\phi} \frac{l_{\phi} F_{E_{2}^{\phi, \bar{\phi}}}}{8-2 \phi(2)} .
$$

The modular symbol $\mathcal{E}=\sum_{g \in \mathbb{P}^{1}\left(\mathbb{Z} / p^{2} \mathbb{Z}\right)} G_{E}(g) \zeta(g) \in \mathrm{H}_{1}\left(X_{0}\left(p^{2}\right)\right.$, cusps, $\left.K\right)$ satisfies $\mathcal{E} \circ c=\pi_{E}(c)$ for all $c \in \mathrm{H}_{1}\left(Y_{0}\left(p^{2}\right), K\right)$.

We now recall the definition of the winding element. Let $\{0, \infty\}$ denote the projection of the path from 0 to $\infty$ in $\mathbb{H} \cup \mathbb{P}^{1}(\mathbb{Q})$ to $X_{0}(N)(\mathbb{C})$. We have an isomorphism $\mathrm{H}_{1}\left(X_{0}(N), \mathbb{Z}\right) \otimes \mathbb{R}=\operatorname{Hom}_{\mathbb{C}}\left(\mathrm{H}^{0}\left(X_{0}(N), \Omega^{1}\right), \mathbb{C}\right)$. Let $e_{N} \in \mathrm{H}_{1}\left(X_{0}(N), \mathbb{Z}\right) \otimes \mathbb{R}$ correspond to the homomorphism $\omega \rightarrow \int_{0}^{\infty} \omega$. The element $e_{N}$ is called the winding element. The explicit expression of $e_{p}$ is the key tool in the proof of the uniform boundedness theorem [10]. Since the integrals of the holomorphic differentials over the Eisenstein element $\mathcal{E}_{2}$ are zero, we write

\section{Corollary 4.10.}

$$
\frac{p^{2}-1}{4} e_{p^{2}}=-\sum_{x \in\left(\mathbb{Z} / p^{2} \mathbb{Z}\right)^{*}} F_{E_{2}}((1,-x))\left\{0, \frac{1}{x}\right\} .
$$

We use Proposition 3.2 to simplify the function $F_{E_{2}}$. We hope that the explicit expression of the winding element will help us to study the Eisenstein ideals [7] of the Hecke algebra for $N=p^{2}$.

\section{ACKNOWLEDGEMENTS}

The author would like to thank Professor Loïc Merel for suggesting the problem and for constant encouragement, patience and several valuable suggestions during the course of the work. He would also like to thank the ARCUS program (French Foreign Ministry/Région Ile-de-France) for giving him an opportunity to visit ParisSud-11.

\section{REFERENCES}

[1] A. Agashe, The special L-value of the winding quotient of level a product of two distinct primes, http://www.math.fsu.edu/agashe/math.html.

[2] Lev A. Borisov and Paul E. Gunnells, Toric modular forms and nonvanishing of L-functions, J. Reine Angew. Math. 539 (2001), 149-165, DOI 10.1515/crll.2001.071. MR.1863857 (2002h:11042)

[3] Fred Diamond and Jerry Shurman, A first course in modular forms, Graduate Texts in Mathematics, vol. 228, Springer-Verlag, New York, 2005. MR2112196 (2006f:11045) 
[4] Albrecht Dold, Lectures on algebraic topology, Classics in Mathematics, Springer-Verlag, Berlin, 1995. Reprint of the 1972 edition. MR 1335915 (96c:55001)

[5] G. Ligozat, Courbes modulaires de genre 1, Publication Mathématique d'Orsay, No. 757411 , U.E.R. Mathématique, Université Paris XI, Orsay, 1974. MR0422158 (54 \#10150)

[6] Ju. I. Manin, Parabolic points and zeta functions of modular curves (Russian), Izv. Akad. Nauk SSSR Ser. Mat. 36 (1972), 19-66. MR0314846 (47 \#3396)

[7] B. Mazur, Modular curves and the Eisenstein ideal, Inst. Hautes Études Sci. Publ. Math. 47 (1977), 33-186 (1978). MR488287 (80c:14015)

[8] B. Mazur, On the arithmetic of special values of L functions, Invent. Math. 55 (1979), no. 3, 207-240, DOI 10.1007/BF01406841. MR553997 (82e:14033)

[9] Loïc Merel, L'accouplement de Weil entre le sous-groupe de Shimura et le sousgroupe cuspidal de $J_{0}(p)$ (French), J. Reine Angew. Math. 477 (1996), 71-115, DOI 10.1515/crll.1996.477.71. MR.1405312 (97f:11045)

[10] Loïc Merel, Bornes pour la torsion des courbes elliptiques sur les corps de nombres (French), Invent. Math. 124 (1996), no. 1-3, 437-449, DOI 10.1007/s002220050059. MR1369424 (96i:11057)

[11] Glenn Stevens, Arithmetic on modular curves, Progress in Mathematics, vol. 20, Birkhäuser Boston Inc., Boston, MA, 1982. MR670070 (87b:11050)

[12] Glenn Stevens, The cuspidal group and special values of L-functions, Trans. Amer. Math. Soc. 291 (1985), no. 2, 519-550, DOI 10.2307/2000098. MR800251 (87a:11056)

[13] Helena A. Verrill, Transportable modular symbols and the intersection pairing, Algorithmic number theory (Sydney, 2002), Lecture Notes in Comput. Sci., vol. 2369, Springer, Berlin, 2002, pp. 219-233, DOI 10.1007/3-540-45455-1_18. MR2041086 (2005b:11063)

Mathematical Sciences Institute, Australian National University, Canberra ACt 0200, Australia

Current address: Indian Institute of Science Education and Research, Dr. Homi Bhabha Road, Pashan, Pune 411 008, India

E-mail address: debargha.banerjee@gmail.com 